\title{
The positive RNASEH1-AS1/has-miR-218-5p/NET1 feedback loop mediated by POU2F1 contributes to the development and progression of human lung squamous carcinoma
}

\section{Jing-hao Jia}

The Fourth Hospital of Hebei Medical University

Jing Wang

North China University of Science and Technology Affiliated People's Hospital

Jia-rui Yu

North China University of Science and Technology Affiliated People's Hospital

\section{Peng Gao}

North China University of Science and Technology Affiliated People's Hospital

Yan-kun Liu

North China University of Science and Technology Affiliated People's Hospital

\section{Yu-feng Li}

North China University of Science and Technology Affiliated People's Hospital

\section{Guo-gui Sun}

North China University of Science and Technology Affiliated People's Hospital

Gao-feng Shi ( $\nabla$ shigaofeng1963@163.com )

The Fourth Hospital of Hebei Medical University

Research article

Keywords: LUSC, IncRNA, RNASEH1-AS1, has-miR-218-5p, NET1, POU2F1

Posted Date: December 14th, 2020

DOI: https://doi.org/10.21203/rs.3.rs-18125/v2

License: (c) (i) This work is licensed under a Creative Commons Attribution 4.0 International License. Read Full License 


\section{Abstract}

\section{Background}

At the molecular level, competing endogenous RNAs (ceRNAs) regulate other RNA transcripts by competing for shared microRNAs (miRNA). Notably, miRNAs negatively regulate gene expression at the levels of mRNA stability and translation suppression.

\section{Methods}

We measured theexpression of miR-218-5p and RNASEH1-AS1 in clinical lung squamous cell carcinoma tissues using qRT-PCR. In an attempt to explore the roles of miR-218-5p and RNASEH1-AS1 in determining the malignant phenotype of $\mathrm{NCl}-\mathrm{H} 520$ cells, colony formation and MTT assays were performed to measure cell viability and proliferation, and transwell invasion and wound healing assays were performed to examine cell migration and invasion. A ChIP assay was conducted to confirm the direct binding of POU2F1 to the RNASEH1-AS1 promoter.

\section{Results}

In this investigation, the expression of the IncRNA RNASEH1-AS1 is upregulated in human lung cancer tissues, and it functions as a miRNA sponge for hsa-miR-218-5p in human lung squamous carcinoma cells. The IncRNA RNASEH1-AS1 facilitates the growth and motility of lung squamous carcinoma cells, while miR-218-5p exerts the opposite effects. NET1 and POU2F1 are validated as direct and functional targets of miR-218-5p. The downregulation of miR-218-5p releases the suppression of NET1 and POU2F1. POU2F1 binds directly to the IncRNA-RNASEH1-AS1 promoter and functions as transcription factor to enhance the promoter activity of RNASEH1-AS1.

\section{Conclusions}

Overall, the positive RNASEH1-AS1/hsa-miR-218-5p/NET1/POU2F1 feedback loop can help us understand the regulatory mechanism underlying the genesis and progression of human lung squamouscarcinoma, possibly providing new biomarkers for its diagnosis and treatment.

\section{Background}

Lung cancer is the most regular and fatal cancer worldwide[1]and is recognized as a cluster of distinct diseases with high levels of genetic, cellular and molecular heterogeneity[2-4]. In general, $80-85 \%$ of human lungcancers are non-small cell lung cancer (NSCLC), of which lung adenocarcinoma (LUAD) and lung squamous carcinoma (LUSC) are the mainsubtypes. Moreover, the genetic and epigenetic alterations differ substantially between LUSC and LUAD. The exploitation ofdrugstargeting particular gene mutations hasdramatically improved the treatment forpatients with advanced LUAD. However, only asmall number of LUSC specimenscontains driver gene mutations, leadingto anextremely low five-year survival ratebecause of compromised efficacy of platinum-based chemotherapy inpatients with LUSC[5]. Thus, 
studies aimingto further address the molecular mechanisms underlying thepathogenesis of LUSC for more effective therapeutic options are crucial.

\section{Recent investigations have unexpectedly discovered a large group consisting of 70 transcripts of non- protein-coding RNAs (ncRNAs) in mammalian cells [6-9]. In contrast, small ncRNAs, such as miRNAs,} siRNAs and piRNAs, longncRNAs (IncRNAs) are a new class of mRNA-like transcripts with sizelonger than 200 nucleotides[10]. Mature miRNAs regulate the expression of most protein-coding genes by binding to the 3'-UTR of target genes, thereby leading to the degradation of target mRNA and suppression of protein translation [11, 12]. In contrast, IncRNAs function as molecular decoys for miRNAs in the cytoplasm and cell nucleus $[13,14]$. The dysregulation of IncRNAs has been reported in many types of cancers, along with its importance in key cancer signalling networks and malignantbehaviours[15], such as in prostate cancer [16], breast cancer [17] and liver cancer [18, 19]. Further studiesexamining the IncRNAs and miRNAs that are dysregulated in LUSCare still urgently needed. LncRNAs were expressed at high levels in lung cancer in our early stage screen. However, the role of RNASEH1-AS1 in LUSC progression and the possible target miRNAs have not been extensively investigated in previous reports. We conducted the in vitro cell experiment and in vivo xenograft assay to explore the effect of RNASEH1-AS1 on LUSC. Notably, miR-218 functions as a IncRNA target and influences the malignant behaviours of many cancer cells, such as hepatocellular carcinoma [20], pancreatic cancer[21] and breast cancer [22]. The expression of miR-218-5p was suppressed in clinical LUSC tissues.

POU2F1(POU class 2 homeobox 1 ) is also known as OCT1, OTF1 or oct-1B. The POU2F1 transcription factor was among the first identified members of the POU transcription factor family [23]. It is a ubiquitous transcription factor that regulates the transcription of genes involved in inflammation and the cell cycle by binding to cis-acting octamer elements[24]. In our study, we investigated the effect of miR218-5p on POU2F1 expression and the relationship between POU2F1 and RNASEH1-AS1 expression in LUSC cells. The NET1 (neuroepithelial cell transforming 1) gene is part of the family of Rho guanine nucleotide exchange factors. The protein encoded by this gene interacts with RhoA within the cell nucleus and may play a role in repairing DNA damage after exposure to ionizing radiation.NET1 is reported to be overexpressed in many human cancers, including non-small cell lung cancer [25-27]. We further examined its expression and its upstream regulatory network in LUSC.

\section{Methods}

\section{Bioinformatics predictions and screening.}

The long non-coding RNAs that areexpressed at high levels in lung cancer were screened using StarBase V3.0 (http://starbase.sysu.edu.cn/).The miRNAsthat bind to the IncRNAs and the possible downstream targets of miR-218-5p were predicted by the most frequently used algorithms TargetScan, miRDB and PicTar, and we noticed the overlappingmiRNAs and targets identified by the three algorithms. The putative promoter of miR-218-5p was predictedusing Promoter Scan (http://wwwbimas.cit.nih.gov/molbio/proscan/). 


\section{Cell line and human LUSC tissue}

$\mathrm{NCl}-\mathrm{H} 520$ cells were cultured with the RPMI-1640 (Gibco, Grand Island, NY, USA) containing $10 \%$ foetal bovine serumand $1,000 \mathrm{U} / \mathrm{mL} \mathrm{P} / \mathrm{S}$. The human LUSC cells were cultured at $37^{\circ} \mathrm{C}$ in a humidified atmosphere with $5 \% \mathrm{CO}_{2}$. Cells were transfectedusing Lipofectamine 2000 Reagent (Invitrogen, USA) according to the manufacturer's instructions. The NCl-H520 cells and three pairs of human lung squamous cell carcinoma tissues were provided by the Department of Cancer Institute, North China University of Science and TechnologyAffiliated People's Hospital. The number, gender, classification and age of the patients with LUSC are shown in Table 1. Informed consent was obtained from all subjects or their direct relatives. The cell and tissue studies weresubmitted to and approved by both the Ethics Committee of North China University of Science and Technology and the Ethics Committee of Hebei Medical University.

\section{qRT-PCR.}

Total RNA was extracted from lung cancer tissues and the cell line with the mirVana miRNA Isolation Kit (Ambion, USA) according to the manufacturer's instructions. Onemicrogram of RNA was reverse transcribed into cDNAswith Moloney murine leukaemia virus reverse transcriptase (Takara, Japan). qRTPCR was conducted with a SYBR ${ }^{B}$ Taq $^{\text {TM }}$ kit (Takara Bio, Japan) and the iQ5 Real Time PCR Detection System. The level of hsa-miR-218-5p in the transcripts was normalized to U6 as the internal control. The levels of the RNASEH1-AS1, NET1 and POU2F1 mRNAs were normalized to $\beta$-actin as the internal control. The quantity of the negative control group was defined as 1 , and the relative fold change of the experimental group was calculated. All the RT and qPCR primer sequences are listed in the Table 2.

\section{Plasmid and miRNA mimics.}

The miR-218-5p overexpression mimics and ASO-miR-218-5pplasmids were commercially synthesized. The sequencesare listed in Table 2.The sh-RNASEH1-AS1 and sh-POU2F1 pSilencer vectors were generated by annealing the sense and antisense strands of the hairpin RNA followed by insertion onto the pSilencer2.1-U6 neo vector between the BamHI and HindllI sites.

The wild type and mutant 3'-UTRs of the NET1 or POU2F1 genesharbouring the predicted miR-218-5p binding site were inserted into the 3 ' end of the reporter gene of the pmirGLO vector. Similarly, the putative promoter area and miR-218-5p binding site in Inc-RNASEH1-AS1 were amplified by PCR and inserted into the 3 ' end of the reporter gene of the pGL3-basic-luciferase vector.

All the insertions mentioned above were verified by DNA sequencing. All primers used in this study are listed in Table 2.

\section{Dual-luciferase reporter assay.}


$\mathrm{NCl}-\mathrm{H} 520$ cells were cultivated in 48-well plates at a density of $6 \times 10^{4}$ cells per well. The miR-218-5p transfection reagents were prepared at a final concentration of $20 \mu \mathrm{M}$ and incubated for $4 \mathrm{~h}$. After $24 \mathrm{~h}$, pmirGLO-targetgene-3'UTR/mut transfection regents were added into cultured cells at a final concentration of $0.5 \mu \mathrm{g}$. After transfection for $4 \mathrm{~h}$, the transfection mixture was replaced with $300 \mu \mathrm{L}$ of fresh complete 1640 medium. After cotransfection for 48 hours, the luciferase activity was measured usingthe Dual-Luciferase Reporter System (Thermo, USA) according to the manufacturer's instructions.

\section{Cell viability and proliferation assays.}

In the MTT assay, $\mathrm{NCl}-\mathrm{H} 520$ (1000 per well) cells were plated into 96 -well plates. At 24,48 , and $72 \mathrm{~h}$ after transfection, MTT was added to every well, and the plates were incubated for $4 \mathrm{~h}$. The absorbance was measured at a wavelength of $570 \mathrm{~nm}$ to evaluate cell viability.

In the colony formation assay, transfected $\mathrm{NCl}-\mathrm{H} 520$ cells (200 cells per well) were trypsinized, plated into 12 -well plates and cultured for 7 to 14 days at $37^{\circ} \mathrm{C}$. The colonies were stained with a solution composed of $0.2 \%$ crystal violet and $20 \%$ methanol. Colonies with greater than 50 cells were counted and analysed. The colony formation rate was calculated using the following formula: colony formation rate = (number of colonies/number of seeded cells) $\times 100 \%$. All cell experiments were conducted at least three times.

\section{Transwell invasion and wound healing assays.}

In the transwell invasion assay, $\mathrm{NCl}-\mathrm{H} 520$ cells $\left(1 \times 10^{5}\right.$ per well) were seeded in the upper chamber of every insert (Millipore, USA) containing $50 \mu \mathrm{l}$ of Matrigel (Millipore, USA). Eight hundred microliters of DMEM supplemented with $20 \%$ FBS (JIBCO, USA) were added to the lower chambers. After $72 \mathrm{~h}$, cells that had attached to the lower surface were stained $\mathrm{f}$ with crystal violet or $15 \mathrm{~min}$. Afterwards, we captured images and counted the cells.

In the wound-healing assay, $\mathrm{NCl}-\mathrm{H} 520$ cells were cultured in 12-well plates. When cell confluence reached $70-80 \%$, scratches were generated with a $50 \mu \mathrm{l}$ pipette tip, and non-adherent cells were removed by three washes with PBS. Wounded cells were cultured in medium lacking FBS for 0,24 , and $48 \mathrm{~h}$. Images of three randomly selected fields of view were captured in each well.

\section{Western blotting.}

$\mathrm{NCl}-\mathrm{H} 520$ cells were collected and lysed with lysis buffer (100 mM Tris-HCl, 2\% SDS, 1 mM mercaptoethanol and $25 \%$ glycerol). Cell extracts were heated in loading buffer and the same amounts of cell extracts were separated on a 10\% SDS-PAGE gels. After electrophoretic transfer to PVDF membranes (Millipore, USA), the protein bands were probed with its corresponding primary antibodies (anti-NET1, antiPOU2F1, Saier Biotechnology, China)overnight at $4{ }^{\circ} \mathrm{C}$. The secondary antibody (anti-GAPDH, Saier Biotechnology, Tianjin, China) was added and incubated with the membrane at room temperature for 1.5 hour. The PVDF membranes were washed with PBS four times and the immunoreactive target bands 
were visualized using the chemiluminescence imaging system (Huqiu Image Instruments, Suzhou, China). Band intensities were quantified using LabWorks image analysis software (UVP, USA).

\section{Animal model.}

Twenty BALB/c-nu mice (female) aged 5 to 6 weekswere purchased from the Institute of Experimental Animals, Chinese Academy of Medical Sciences (Beijing). They were randomly divided into two groups.In total, $2 \times 10^{7} \mathrm{NCl}-\mathrm{H} 520$ cells were transfected with siR-RNASEH1-AS1 or siR-NC and suspended in $100 \mu \mathrm{l}$ of serum-free RPMI1640 for every nude mouse. The cell suspension was injected directly into the left side of the back of the mice. The tumour volume was measured every three days after injection. Four weeks later, anaesthetized mice were sacrificed by cervical dislocation, and their tumourmasseswere harvested. No premature deaths were documented. The tumour weight was measured and the average tumour weight was calculated. The tumour tissues were stored at $-80^{\circ} \mathrm{Cor}$ used to perform haematoxylin-eosin (HE) and immunohistochemical staining.All studies were performed under the American Association for the Accreditation of Laboratory Animal Care guidelines and adhered to national and international standards. All animal experiments were approved by the Ethics Committee of Hebei Medical University.

\section{CHIP assay.}

The binding of POU2F1 to the Inc-RNASEH1-AS1 promoter was confirmed by ChIP assay according to the instructions of Chromatin Immunoprecipitation Kit (Millipore, USA). NCl-H520 cells were used in the crosslinking step after reaching $80-90 \%$ confluence. Isolated chromatin was sonicated to shear the DNA. Next, immunoprecipitation and elution of cross-linked protein/DNA were performed according to the manufacturers' protocol. Three to five micrograms of anti-RNA polymerase or anti-Rabbit IgG were used as the positive and negative control groups, respectively. Three to five microgramsof anti-POU2F1was used in the experimental group. Cross-links of protein/DNA complexes were reversed to free DNA, and spin columns were used for DNA purification. Primers flanking the predicted POU2F1 binding site in the Inc-RNASEH1-AS1 promoter were used for PCR. The primers used in this studyare listed in Table 2.The LabWorks image acquisition and analysis system (UVP, USA) was used to capture images and quantify the intensities of target signals after gel electrophoresis. The quantity of the input group or anti-POU2F1ChIP group was 3481 or 8041 , respectively. The quantity of the input group was defined as 1 , and the fold change (2.30) in the anti-POU2F1-ChIP group was calculated.

\section{Statistical evaluation.}

The data were analysed using GraphPad Prism 6 Software (GraphPad Software, USA) with the two-tailed Student's $t$ test. The results are presented as the means \pm S.D. of three separate experiments. Unpaired Student's t-test was used to compare the two groups. A p value less than 0.05 was regarded as a

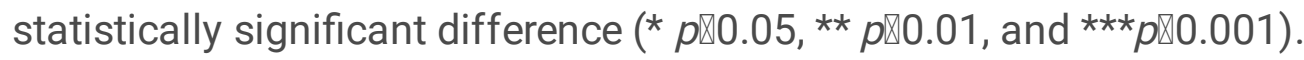

\section{Results}




\section{The IncRNA RNASEH1-AS1 is expressedat high levels and functions as a miRNA sponge for miR-218-5p in human lung cancer.}

We used StarBase V3.0 to identify IncRNAs that are dysregulated in human lung cancer tissues. The screening results revealed high RNASEH1-AS1 expression in 526 cancer samples compared to 59 normal samples (Fig.1A). We alsodetected theexpression level of the RNASEH1-AS1 mRNA in three pairs of clinical lung squamous cell carcinoma tissues, and RNASEH1-AS1 was expressedat higher levels in LUSC tissues than in theadjacent normal tissues (Fig. 1B). Base-pairing complementation showed that IncRNASEH1-AS1 contains a putative binding sitewithobvious complementarity to the seed region of miR218-5p (Fig. 1C). When wild typelncRNA RNASEH1-AS1 was cotransfected with miR-218-5p mimics or ASO-218-5p, the luciferase activity of $\mathrm{NCl}-\mathrm{H} 520$ cells was obviously reduced or increased, respectively, compared withtheir corresponding controls (Fig. 1C). However, both overexpression and inhibition of miR218-5p did not exert an apparent effect on the luciferase intensity of cells transfected with the IncRNASEH1-AS1mutant (Fig. 3C).

The level of the RNASEH1-AS1 mRNA was measured using qRT-PCR to assess the effect of miR-218-5p on endogenous IncRNA RNASEH1-AS1 expression.The expression of RNASEH1-AS1 was reduced upon miR-218-5p overexpression (Fig. 1D). Meanwhile, a small interfering RNA (siRNA) for RNASEH1-AS1 was synthesized, and the effect of pSilencer/shRNA-RNASEH1-AS1 was verified using qRT-PCR (Fig. 1E). Knockdown of RNASEH1-AS1 resulted in 2.9-fold increases in the endogenous miR-218-5p level in NClH520 cells (Fig. 1F).In contrast to the IncRNA RNASEH1-AS1, miR-218-5p was expressed at lower levels in clinical lung squamous cell carcinoma samples than in adjacent normal tissue samples(Fig. 1G).

\section{miR-218-5p suppresses the proliferation and motility of LUSC cells in vitro, while IncRNA-RNASEH1-AS1 exerts the opposite effects.}

We transfected the miR-218-5p overexpression construct and ASO mimic into $\mathrm{NCl}-\mathrm{H} 520$ cells and performed some functional experiments to explore the effect of miR-218-5p on the malignant phenotype of LUSC cells. In the MTT assay, miR-218-5p obviously reduced the OD value of NCI-H520 cells at 48 and 72 hours after transfection, while its ASO mimicsincreased cell viability at 24,48 and 72 hours(Fig. 2A). As shown in the colony formation assay, miR-218-5p apparently decreased the colony formation rate of $\mathrm{NCl}-\mathrm{H} 520$ cells, while its ASO mimics increased the colony formation rate compared to its corresponding control (Fig. 2C). In other words, miR-218-5p inhibited both the viability and the growth of LUSC cells in vitro.

We also conducted transwell invasion and wound-healing assaysusing $\mathrm{NCl}-\mathrm{H} 520$ cells to investigate the function of miR-218-5p in cell motility. The data revealed that miR-218-5p reduced the number of invasive cells (Fig. 2E) and enlarged the distance of cell gaps at 48 hours (Fig. 2G) compared with the control group, while its ASO mimic exerted the opposite effects (Fig. 2E and 2G). Our study focused on IncRNASEH1-AS1 at the same time. Theviability (Fig. 2B), growth (Fig. 2D), migration (Fig. 2G) and invasion (Fig. 2F) of NCl-H520 cells was suppressed after Inc-RNASEH1-AS1 interference. The miR-218-5p ASO 
mimics counteracted the inhibitory effect of sh-RNASEH1-AS1 on the growth of LUSC cells (Fig. 2D), but did not result in significance differencesin cell viability, migration and invasion (Fig. 2B, 2F and 2G).

\section{NET1 and POU2F1 were identified as direct targets of miR-218-5p.}

We screened the potential downstream targets of miR-218-5p using the TargetScan, miRDB and PicTar databases to determine the mechanism by which miR-218-5p regulates the oncogenesis of lung squamous carcinoma cells (Fig. 3A). Among the overlapping target genes, NET1 and POU2F1 were selected for further investigation.

The 3' UTRs of both NET1 and POU2F1 contain putative sites that are conserved among many species and displayapparent complementarity with the seed region of miR-218-5p, according to the base-pairing rules (Fig. 3B and 3C). The 3'UTRs of NET1 or POU2F1 was co-transfectedwith miR-218-5p into NCl-H520 cells. The transfection of miR-218-5p reduced the fluorescence intensity, while the transfection ofits ASO mimicsincreased the fluorescence intensity in the dual-luciferase reporter assay (Fig. 3B and 3C). In contrast, neither the overexpression nor knockdown of miR-218-5p exerted an obviouseffect on the fluorescence intensity of $\mathrm{NCl}-\mathrm{H} 520$ cells transfected with constructs containing the $3^{\prime} \mathrm{UTR}$ of NET1 or POU2F1 with the mutated miR-218-5p binding sequence (Fig. 3B and 3C). These results revealed that miR218-5p directly binds to the 3'-UTR of NET1 and POU2F1.According to the qRT-PCR results, miR-218-5p downregulatedthe expression of the endogenous NET1 and POU2F1 mRNAs in NCl-H520 cells (Fig. 3D). Western blot analysis revealed that miR-218-5papparently reduced the levelsofthe endogenous NET1 and POU2F1 proteins (Fig. 3E and 3F), indicating that miR-218-5p negatively regulates NET1 and POU2F1 expression by binding to their $3^{\prime}$-UTRs.

Wesubsequently examined the expression of the NET1 mRNA in three pairs of clinical lung squamous cell carcinoma samples. As expected, NET1 was expressed at higherlevels in LUSC tissues than in corresponding normal tissues (Fig. 3G), in contrast to miR-218-5p and similar to Inc-RNASEH1-AS1.

\section{Downregulation of IncRNA-RNASEH1-AS1 inhibitsthe growth of tumours formed by LUSC cells in vivo.}

We conducted a tumourigenicity assayin nude mice to assess the effect of the IncRNA RNASEH1-AS1 in vivo. Twenty mice were randomly divided into two groups of ten mice per group. $\mathrm{NCl}-\mathrm{H} 520$ cells transfected with siR-RNASEH1-AS1 or siR-NC were subcutaneously injected into the left back of the nude mice. Figure 4A shows images of the two groups of nude mice at 4 weeks after injection, and figure $4 B$ shows images of the tumour masses after the animals were sacrificed. The tumoursgrew slower in nude micefrom the Inc-RNASEH1-AS1 interference group than the control group (Fig. 4C), and the average tumour volume of the Inc-RNASEH1-AS1 interference group was smaller thanthe control group (Fig.4D). The weight of the tumour mass was lighter than the control group (Fig. 4E).Figure 4F shows the loci oftumours derived from transfected cellsdetected using haematoxylin-eosin (HE)staining and NET1 or POU2F1expression detected using immunohistochemical staining. NET1 and POU2F1 expression were significantly reduced after RNASEH1-AS1 interference, as evidenced by the immunohistochemical staining (Fig. 4F).The expression of miR-218-5p was apparently increased and the expression of the 
targets of miR-218-5p were decreased in xenograft tumours after RNASEH1-AS1 interference, as evidenced by the qRT-PCR results (Fig. 4G). Moreover, the protein levels of its targets were also decreased,as determined using westernblotting (Fig. 4H).Based on these data, the downregulation of the IncRNA RNASEH1-AS1 promoted the upregulation of miR-218-5p and downregulation of its target genes and suppressed the growth of lung squamous carcinomacells in vivo.

\section{POU2F1 binds directly to the IncRNA-RNASEH1-AS1 promoter and stimulates its promoter activity.}

We constructed a POU2F1 interfering plasmid and validated its efficiency using qRT-PCR to verify whether POU2F1 is a potential transcription factor for RNASEH1-AS1 (Fig. 5A). As shown in figure 5A and 5B, knockdown of POU2F1 effectively reduced the expression and promoter luciferase activity of RNASEH1AS1. A ChIP assay was conducted with an anti-POU2F1 antibody using NCl-H520 cell lysates, followed by PCR with primers designed toamplify the RNASEH1-AS1 promotor. The intensity of target signal was higher than the input (Fig. 5C and 5D). The band indicated the direct interact and positive binding of POU2F1 to the RNASEH1-AS1 promoter. Figure 5E shows a schematic of the regulatory mechanism of the IncRNARNASEH1-AS1/hsa-miR-218-5p/NET1/POU2F1 axis in human lung squamous carcinoma cells.

\section{Discussion}

Recently, researchers have discovered that the interaction of the miRNA seed sequence with mRNA is not unidirectional, and the pool of pseudogenes, mRNAs, long non-coding RNAs (IncRNAs), circular RNAs (circRNAs) compete for the same pool of miRNAs $[28,29]$. These competitive endogenous RNAs (ceRNAs) serve as molecular sponges for miRNAs by interacting with their miRNA binding sites, consequently derepressing all targets of the corresponding miRNA family. An increasing number of researchers have paid close attention to IncRNAs as competing endogenous RNAs for miRNAs during tumourigenesis and progression in recent years. Numerous IncRNAs are abnormally expressed or mutated in many types of cancers [30]. HULC was upregulated in both tumours and plasma from patients with hepatocellular carcinoma and was a possible biomarker for HCC [31].Abnormal X chromosome inactivation caused by aberrantly expressed XIST promotes carcinogenesis in leukaemia[32]. PCA3 is over-expressed in ninetyfive percent of prostate cancer clinical samples and is a marker with high specificity in the urine of patients with benign and malignant prostate cancer $[33,34]$. In ourstudy, we firstdiscovered that the InCRNARNASEH1-AS1 was expressed at high levels in 526 lung cancer samples compared to 59 normal tissue samples by screening StarBase and was expressed at higher in LUSC tissues than in the adjacent normal tissues. Moreover, the viability, proliferation, invasion and migration of $\mathrm{NCl}-\mathrm{H} 520$ cells were reduced after RNASEH1-AS1 interference, suggesting the oncogenic character of RNASEH1-AS1 in LUSC. We observed the same oncogenic effect of RNASEH1-AS1 on LUSC in the in vivo xenograft animal model and in vitro cell experiment.

Our study also discovered that RNASEH1-AS1 functions as a ceRNA by directly interacting with the seed region of miR-218-5p in LUSC samples. The expression of miR-218-5p in LUSC cells was suppressed due to the upregulation of RNASEH1-AS1. In contrast to RNASEH1-AS1, miR-218-5p inhibited LUSC cell 
growth and motility and functioned as a tumour suppressor gene in lung squamous carcinoma. According to a recent study by Yu et al[35],circRNA-104718 functions as a ceRNA and promotes HCC progression by regulating the microRNA-218-5p-TXNDC5 signalling pathway. Li et al[36]found that oncogenic KSHV-encoded interferon regulatory factor upregulates HMGB2 and CMPK1 to promote cell invasion by disrupting the IncRNA-OIP5-AS1-miR-218-5p network. Ye et al[37] also discovered that the E2F1-mediated MNX1-AS1-miR-218-5p-SEC61A1 feedback loop is involved in the progression of colon adenocarcinoma. Our results expand the recognition of miR-218-5p and its competing endogenous RNA as playing a role in the development and progression of LUSC.

Half of all genes in the genome are estimated to be targets of miRNAs, spanning a large regulatory mechanismat the post-transcriptional level [11]. We identified both NET1 and POU2F1 as direct targets of miR-218-5p. Importantly, miR-218-5p negatively regulated NET1 and POU2F1 expression by directly binding to their 3'-UTRsin LUSC cells. The levels of the NET1 and POU2F1 mRNAs and proteins were decreased in xenograft tumour tissues after RNASEH1-AS1 interference. In other words, downregulation of the IncRNA RNASEH1-AS1 led to the upregulation of miR-218-5p, thereby resulting in the downregulation of its target genes. POU2F1 is also regulated by miR-665 and miR-9-5p in human osteosarcoma[38, 39], and by miR-449 in liver cancer [40]. Our study provides the first evidence that identified POU2F1 and NET1 as the downstream molecules of miR-218-5p and RNASEH1-AS1 in lung squamous cell carcinoma. The RhoA activating protein NET1 makes fundamental contributions to mammary gland tumorigenesis and metastasis [41]. NET1 expression was strongly associated with the patients' pathological characteristics, including clinical stage, lymph node metastasis, distant metastasis and differentiation degree and had a significant role in the tumorigenesis of human non small cell lung cancer [27]. However, the influence of NET1 on the malignant phenotype of lung squamous carcinoma cells have not been discovered in any reports before and needs to be studied in the future.

More importantly, our data revealed that POU2F1 interference attenuates the activity of the RNASEH1-AS1 promoter in LUSC cells. The direct binding of POU2F1 to the RNASEH1-AS1 promoter was validated by the results of the chromatin immunoprecipitation assay. POU2F1 not only represents a downstream target gene of miR-218-5p but also the upstream transcription factor for RNASEH1-AS1.

\section{Conclusion}

Taken together, RNASEH1-AS1 functions as an oncogene by serving as a molecular sponge for miR-218$5 p$. The downregulation of miR-218-5p releases the suppression of NET1 and POU2F1. POU2F1 binds directly to the RNASEH1-AS1 promoter to induce its expression, thereby forming a closedIncRNA-miRNAGENE-TF regulatory loop in LUSC. These findings expand our understanding of the positive feedback loop of RNASEH1-AS1/hsa-miR-218-5p/NET1/POU2F1 and the mechanism underlying the genesis and progression of human lung squamous carcinoma, possibly providing new biomarkers for its diagnosis and treatment.

\section{Abbreviations}


ceRNAs: Competing endogenous RNAs

miRNA: MicroRNA

LncRNA: Long non-coding RNA

CircRNA: Circular RNA

NSCLC: Non-small cell lung cancer

LUSC: Lung squamous cell carcinoma

LUAD: Lung adenocarcinoma

RNASEH1-AS1: RNASEH1 antisense RNA 1

POU2F1: POU class 2 homeobox 1

NET1: Neuroepithelial cell transforming 1

\section{Declarations}

\section{Ethical approval and consent to participate}

All studies were performed according to the American Association for the Accreditation of Laboratory Animal Care guidelines and adhered to national and international standards. Prior to obtaining thetissue specimens, informed consent was obtained from all subjects or their direct relatives.All cell and tissue studies were submitted to and approved by both the Ethics Committee of North China University of Science and Technology and the Ethics Committee of Hebei Medical University. All animal experiments were submitted to and approved by the Ethics Committee of Hebei Medical University.

\section{Consent for publication}

Not applicable.

\section{Availability of data and materials}

The datasets used and/or analysed during the current study are available from the corresponding author upon reasonable request.

\section{Competing interests}

All authors have no conflicts of interest to declare.

\section{Funding}


This work was supported by the Young Top-Notch talent Project of Hebei province (No. JI2016(10), Talent Project of Hebei province (A201801005), Academician Workstation Construction Special Project Of Tangshan People's Hospital (199A77119H), Natural Science Foundation of Outstanding Youth of Hebei Province (H2019105026), and Basic Research Cooperation Project of Beijing-Tianjin-Hebei [H2019105143,19JCZDJC64500(Z)]. No benefits in any form have been or will be received from a commercial party related directly or indirectly to the subject of this study.

\section{Authors' contributions}

GF S designed and supervised the completion of the experiment. GG S designed, directed the experiment and revised the manuscript. $\mathrm{JH} \mathrm{J}$ wrote and revised the manuscript, and was a major contributor in performing the experiments and analyzing data. JW prepared the clinical samples and participated in performing the experiment and manuscript preparation. JR $Y$ analyzed and interpreted the data. PG performed part of the experiment. YK L and YF L participated in the experimental coordination. All authors read and approved the final manuscript.

\section{Acknowledgements}

Not applicable.

\section{References}

1. Siegel R, Ma J, Zou Z, Jemal A: Cancer statistics, 2014. CA: a cancer journal for clinicians 2014, 64(1):9-29.

2. de Bruin EC, McGranahan N, Swanton C: Analysis of intratumor heterogeneity unravels lung cancer evolution. Molecular \& cellular oncology 2015, 2(3):e985549.

3. Cancer Genome Atlas Research N: Comprehensive genomic characterization of squamous cell lung cancers. Nature 2012, 489(7417):519-525.

4. Ding L, Getz G, Wheeler DA, Mardis ER, McLellan MD, Cibulskis K, Sougnez C, Greulich H, Muzny DM, Morgan MB et al: Somatic mutations affect key pathways in lung adenocarcinoma. Nature 2008, 455(7216):1069-1075.

5. Rizvi NA, Hellmann MD, Brahmer JR, Juergens RA, Borghaei H, Gettinger S, Chow LQ, Gerber DE, Laurie SA, Goldman JW et al: Nivolumab in Combination With Platinum-Based Doublet Chemotherapy for First-Line Treatment of Advanced Non-Small-Cell Lung Cancer. Journal of clinical oncology : official journal of the American Society of Clinical Oncology 2016, 34(25):2969-2979.

6. Mattick JS, Makunin IV: Non-coding RNA. Human molecular genetics 2006, 15 Spec No 1:R17-29.

7. Huttenhofer A, Kiefmann M, Meier-Ewert S, O'Brien J, Lehrach H, Bachellerie JP, Brosius J: RNomics: an experimental approach that identifies 201 candidates for novel, small, non-messenger RNAs in mouse. The EMBO journal 2001, 20(11):2943-2953. 
8. Kapranov P, Cawley SE, Drenkow J, Bekiranov S, Strausberg RL, Fodor SP, Gingeras TR: Large-scale transcriptional activity in chromosomes 21 and 22. Science 2002, 296(5569):916-919.

9. Kopp F, Mendell JT: Functional Classification and Experimental Dissection of Long Noncoding RNAs. Cell 2018, 172(3):393-407.

10. Guttman M, Amit I, Garber M, French C, Lin MF, Feldser D, Huarte M, Zuk O, Carey BW, Cassady JP et al: Chromatin signature reveals over a thousand highly conserved large non-coding RNAs in mammals. Nature 2009, 458(7235):223-227.

11. Friedman RC, Farh KK, Burge CB, Bartel DP: Most mammalian mRNAs are conserved targets of microRNAs. Genome research 2009, 19(1):92-105.

12. Chou CH, Shrestha S, Yang CD, Chang NW, Lin YL, Liao KW, Huang WC, Sun TH, Tu SJ, Lee WH et al: miRTarBase update 2018: a resource for experimentally validated microRNA-target interactions. Nucleic acids research 2018, 46(D1):D296-D302.

13. Liu X, Li D, Zhang W, Guo M, Zhan Q: Long non-coding RNA gadd7 interacts with TDP-43 and regulates Cdk6 mRNA decay. The EMBO journal 2012, 31(23):4415-4427.

14. Yuan JH, Yang F, Wang F, Ma JZ, Guo YJ, Tao QF, Liu F, Pan W, Wang TT, Zhou CC et al: A long noncoding RNA activated by TGF-beta promotes the invasion-metastasis cascade in hepatocellular carcinoma. Cancer cell 2014, 25(5):666-681.

15. Bartonicek N, Maag JL, Dinger ME: Long noncoding RNAs in cancer: mechanisms of action and technological advancements. Molecular cancer 2016, 15(1):43.

16. Prensner JR, lyer MK, Balbin OA, Dhanasekaran SM, Cao Q, Brenner JC, Laxman B, Asangani IA, Grasso CS, Kominsky HD et al: Transcriptome sequencing across a prostate cancer cohort identifies PCAT-1, an unannotated lincRNA implicated in disease progression. Nature biotechnology 2011, 29(8):742-749.

17. Su X, Malouf GG, Chen Y, Zhang J, Yao H, Valero V, Weinstein JN, Spano JP, Meric-Bernstam F, Khayat $D$ et al: Comprehensive analysis of long non-coding RNAs in human breast cancer clinical subtypes. Oncotarget 2014, 5(20):9864-9876.

18. Geng YJ, Xie SL, Li Q, Ma J, Wang GY: Large intervening non-coding RNA HOTAIR is associated with hepatocellular carcinoma progression. The Journal of international medical research 2011, 39(6):2119-2128.

19. He Y, Meng XM, Huang C, Wu BM, Zhang L, Lv XW, Li J: Long noncoding RNAs: Novel insights into hepatocelluar carcinoma. Cancer letters 2014, 344(1):20-27.

20. Ji D, Wang Y, Sun B, Yang J, Luo X: Long non-coding RNA MNX1-AS1 promotes hepatocellular carcinoma proliferation and invasion through targeting miR-218-5p/COMMD8 axis. Biochemical and biophysical research communications 2019, 513(3):669-674.

21. Liu S, Zhang W, Liu K, Liu Y: LncRNA SNHG16 promotes tumor growth of pancreatic cancer by targeting miR-218-5p. Biomedicine \& pharmacotherapy = Biomedecine \& pharmacotherapie 2019, 114:108862. 
22. Hu X, Ding D, Zhang J, Cui J: Knockdown of IncRNA HOTAIR sensitizes breast cancer cells to ionizing radiation through activating miR-218. Bioscience reports 2019, 39(4).

23. Sturm RA, Cassady JL, Das G, Romo A, Evans GA: Chromosomal structure and expression of the human OTF1 locus encoding the Oct-1 protein. Genomics 1993, 16(2):333-341.

24. Tantin D, Schild-Poulter C, Wang V, Hache RJ, Sharp PA: The octamer binding transcription factor Oct1 is a stress sensor. Cancer research 2005, 65(23):10750-10758.

25. Shen SQ, Li K, Zhu N, Nakao A: Expression and clinical significance of NET-1 and PCNA in hepatocellular carcinoma. Medical oncology 2008, 25(3):341-345.

26. Dutertre M, Gratadou L, Dardenne E, Germann S, Samaan S, Lidereau R, Driouch K, de la Grange P, Auboeuf $D$ : Estrogen regulation and physiopathologic significance of alternative promoters in breast cancer. Cancer research 2010, 70(9):3760-3770.

27. Fang L, Zhu J, Ma Y, Hong C, Xiao S, Jin L: Neuroepithelial transforming gene 1 functions as a potential prognostic marker for patients with non-small cell lung cancer. Molecular medicine reports 2015, 12(5):7439-7446.

28. Cesana M, Cacchiarelli D, Legnini I, Santini T, Sthandier O, Chinappi M, Tramontano A, Bozzoni I: A long noncoding RNA controls muscle differentiation by functioning as a competing endogenous RNA. Cell 2011, 147(2):358-369.

29. Memczak S, Jens M, Elefsinioti A, Torti F, Krueger J, Rybak A, Maier L, Mackowiak SD, Gregersen LH, Munschauer $\mathrm{M}$ et al: Circular RNAs are a large class of animal RNAs with regulatory potency. Nature 2013, 495(7441):333-338.

30. Bhan A, Mandal SS: Long noncoding RNAs: emerging stars in gene regulation, epigenetics and human disease. ChemMedChem 2014, 9(9):1932-1956.

31. Panzitt K, Tschernatsch MM, Guelly C, Moustafa T, Stradner M, Strohmaier HM, Buck CR, Denk H, Schroeder R, Trauner $M$ et al: Characterization of HULC, a novel gene with striking up-regulation in hepatocellular carcinoma, as noncoding RNA. Gastroenterology 2007, 132(1):330-342.

32. Alvarez-Dominguez JR, Hu W, Gromatzky AA, Lodish HF: Long noncoding RNAs during normal and malignant hematopoiesis. International journal of hematology 2014, 99(5):531-541.

33. Warrick JI, Tomlins SA, Carskadon SL, Young AM, Siddiqui J, Wei JT, Chinnaiyan AM, Kunju LP, Palanisamy N: Evaluation of tissue PCA3 expression in prostate cancer by RNA in situ hybridizationa correlative study with urine PCA3 and TMPRSS2-ERG. Modern pathology : an official journal of the United States and Canadian Academy of Pathology, Inc 2014, 27(4):609-620.

34. Chevli KK, Duff M, Walter P, Yu C, Capuder B, Elshafei A, Malczewski S, Kattan MW, Jones JS: Urinary PCA3 as a predictor of prostate cancer in a cohort of 3,073 men undergoing initial prostate biopsy. The Journal of urology 2014, 191(6):1743-1748.

35. Yu J, Yang M, Zhou B, Luo J, Zhang Z, Zhang W, Yan Z: CircRNA-104718 acts as competing endogenous RNA and promotes hepatocellular carcinoma progression through microRNA-2185p/TXNDC5 signaling pathway. Clinical science 2019, 133(13):1487-1503. 
36. Li W, Wang Q, Feng Q, Wang F, Yan Q, Gao SJ, Lu C: Oncogenic KSHV-encoded interferon regulatory factor upregulates HMGB2 and CMPK1 expression to promote cell invasion by disrupting a complex IncRNA-OIP5-AS1/miR-218-5p network. PLoS pathogens 2019, 15(1):e1007578.

37. Ye Y, Gu B, Wang Y, Shen S, Huang W: E2F1-mediated MNX1-AS1-miR-218-5p-SEC61A1 feedback loop contributes to the progression of colon adenocarcinoma. Journal of cellular biochemistry 2019, 120(4):6145-6153.

38. Jin XM, Xu B, Zhang Y, Liu SY, Shao J, Wu L, Tang JA, Yin T, Fan XB, Yang TY: LncRNA SND1-IT1 accelerates the proliferation and migration of osteosarcoma via sponging miRNA-665 to upregulate POU2F1. European review for medical and pharmacological sciences 2019, 23(22):9772-9780.

39. Xie CH, Cao YM, Huang Y, Shi QW, Guo JH, Fan ZW, Li JG, Chen BW, Wu BY: Long non-coding RNA TUG1 contributes to tumorigenesis of human osteosarcoma by sponging miR-9-5p and regulating POU2F1 expression. Tumour biology : the journal of the International Society for Oncodevelopmental Biology and Medicine 2016, 37(11):15031-15041.

40. Liu Y, Wang Y, Sun X, Mei C, Wang L, Li Z, Zha X: miR-449a promotes liver cancer cell apoptosis by downregulation of Calpain 6 and POU2F1. Oncotarget 2016, 7(12):13491-13501.

41. Zuo Y, Ulu A, Chang JT, Frost JA: Contributions of the RhoA guanine nucleotide exchange factor Net1 to polyoma middle $T$ antigen-mediated mammary gland tumorigenesis and metastasis. Breast cancer research : BCR 2018, 20(1):41.

\section{Tables}

Table 1. Clinical Tissue Samples Used in This Study.

\begin{tabular}{ccccc}
\hline Number & Gender & Classification & Age & Date \\
\hline $\mathbf{1}$ & Male & LUSC & 63 & 2019.2 .26 \\
$\mathbf{2}$ & Male & LUSC & 50 & 2019.3 .6 \\
$\mathbf{3}$ & Male & LUSC & 62 & 2019.4 .29 \\
\hline
\end{tabular}

Table 2. The PCR Primers Used in This Study. 
Isa-miR-

?18-5p-RT

Isa-miR-

:18-5p-Fwd

J6 RT

J6 Forward

Reverse

RNASEH1-

IS1-qPCR-

;

RNASEH1-

IS1-qPCR-

tS

NET1-

IPCR-S

VET1-

IPCR-AS

POU2F1-

IPCR-S

'OU2F1-

IPCR-AS

3-actin-

IPCR-S

3-actin-

IPCR-AS

RNASEH1- TCTGAGGGAGACTGATTC

tS1-Chip-S

RNASEH1-

tS1-Chip-

tS

RNASEH1-

tS1-target-

「op

RNASEH1tS1-target3ot

2NASEH1tS1-targetnut-Top

TGCGGTTGTGCTTGATC
GTCGTATCCAGTGCAGGGTCCGAGGTGCACTGGATACGACACATGGTTAG

GTCGTATCCAGTGCAGGGTCCGAGGTATTCGCACTGGATACGACAAAATATGGAAC

TGCGGGTGCTCGCTTCGGCAGC

CCAGTGCAGGGTCCGAGGT

GAGAAGCACCCGCACCTGG

GCCTCTAATCCCAACACT

GAAAGGTGAATCCGAGTG

GTGCCGTTCGTTCCGTGT

GTAACCGCCGCCAGAAAG

TGGAGGCTGAGGCAGAAGG

CGTGACATTAAGGAGAAGCTG

CTAGAAGCATTTGCGGTGGAC

ACAGGTGTAGAGTCTGACGTGC

AAACTAGGAATTCATGAATTAGAATAAGCACAAAGGAGGGCGAGAGGCT

CTAGAGCCTCTCGCCCTCCTTTGTGCTTATTCTAATTCATGAATTCCTAGTTT

AAACTAGGAATTCATGAATTAGAATCGTACTGCAGGAGGGCGAGAGGCT

Page 16/23 
2NASEH1tS1-targetnut-Bot

VET13’UTR-Top

VET1-

3'UTR-Bot

VET13'UTR-mut-

Гop

VET1 3'UTR-mut3ot

POU2F13’UTR-Top

OUU2F13'UTR-Bot

POU2F13'UTR-mut-

Гop

OUU2F13'UTR-mut3ot

;h-POU2F Гop

;h-POU2F 3ot

jh1 RNASEH1 -

tS1-top

ih1 RNASEH1 tS1-bot

CTAGAGCCTCTCGCCCTCCTGCAGTACGATTCTAATTCATGAATTCCTAGTTT

AAACTAGGAATGATACTATTAAAAAAAAAAAAAGCACACACATAATCACCCTGCT

CTAGAGCAGGGTGATTATGTGTGTGCTTTTTTTTTTTTAATAGTATCATTCCTAGTTT

AAACTAGGAATGATACTATTAAAAAAAAAAACGTACTGCACATAATCACCCTGCT

CTAGAGCAGGGTGATTATGTGCAGTACGTTTTTTTTTTAATAGTATCATTCCTAGTTT

AAACTAGGTTGGGGGAAAAAAAAGCACAACTATACCTCTTTAATGTTATTTTCCT

CTAGAGGAAAATAACATTAAAGAGGTATAGTTGTGCTTTTTTTTCCCCCAACCTAGTTT

AAACTAGGTTGGGGGAAAAAACGTACTGCCTATACCTCTTTAATGTTATTTTCCT

CTAGAGGAAAATAACATTAAAGAGGTATAGGCAGTACGTTTTTTCCCCCAACCTAGTTT

GATCCCCAGTCAACACCAAAGCGAATCTCGAGATTCGCTTTGGTGTTGACTGGTTTTTGA AGCTTCAAAAACCAGTCAACACCAAAGCGAATCTCGAGATTCGCTTTGGTGTTGACTGGG GATCCGAGAAGCACCCGCACCTGGAGCTCGAGCTCCAGGTGCGGGTGCTTCTCTTTTTGA AGCTTCAAAAAGAGAAGCACCCGCACCTGGAGCTCGAGCTCCAGGTGCGGGTGCTTCTCG

\section{Figures}


A

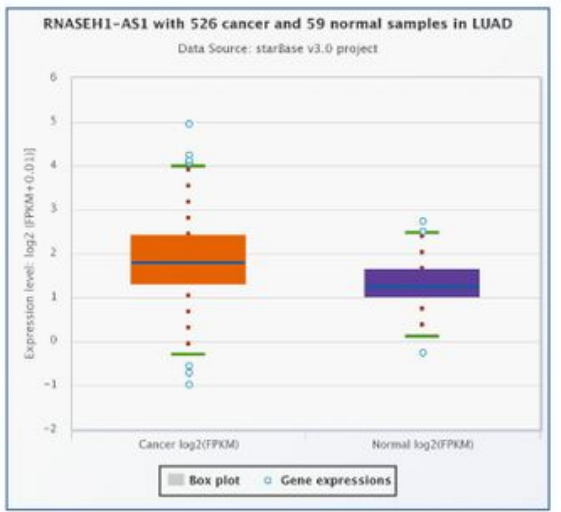

C

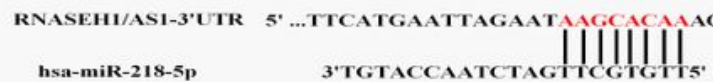

RNASEH1/AS1-3'UTR-mut 5'...TTCATGAATTAGAATCGTACTGCAGGA...3*

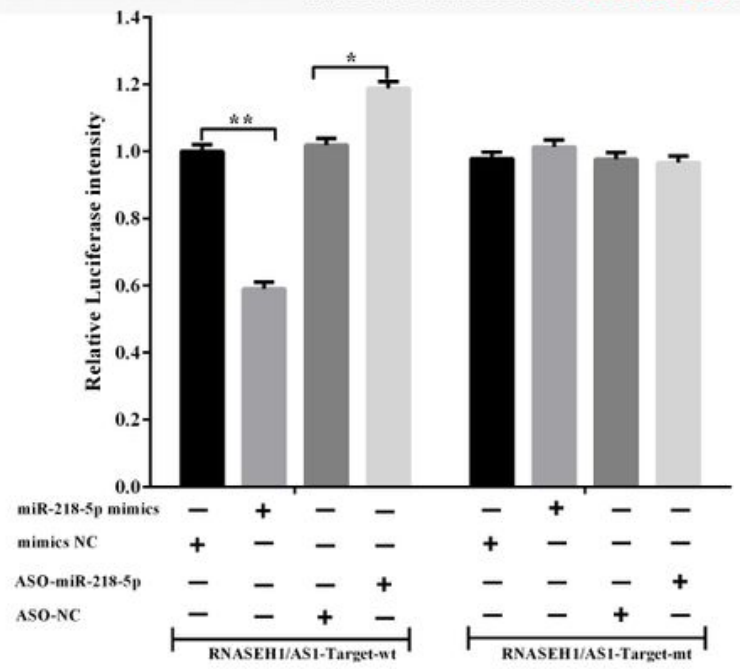

B

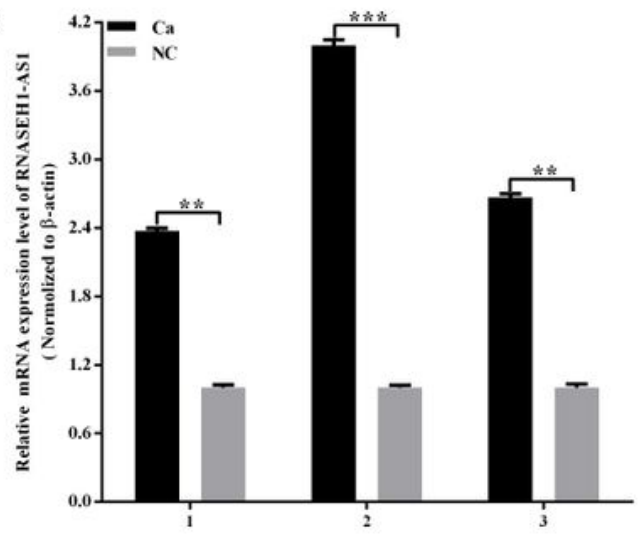

D

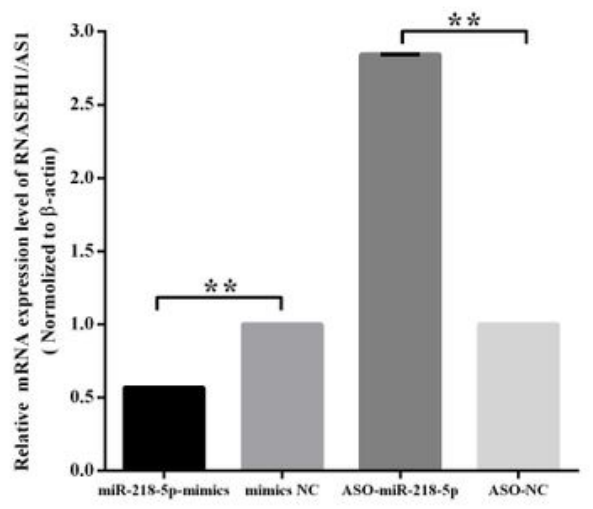

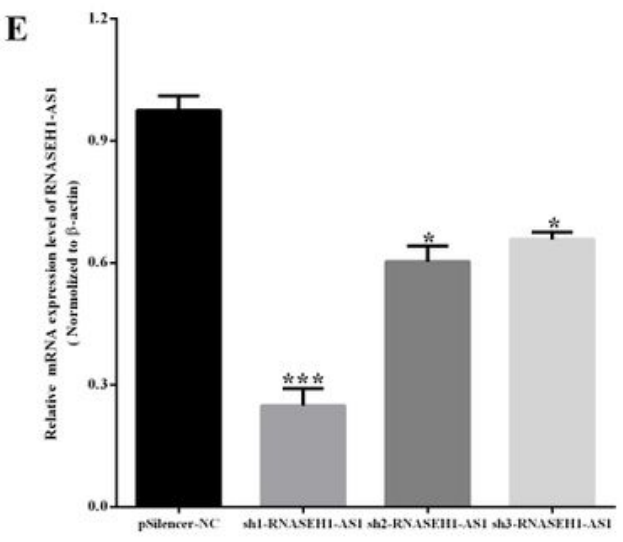
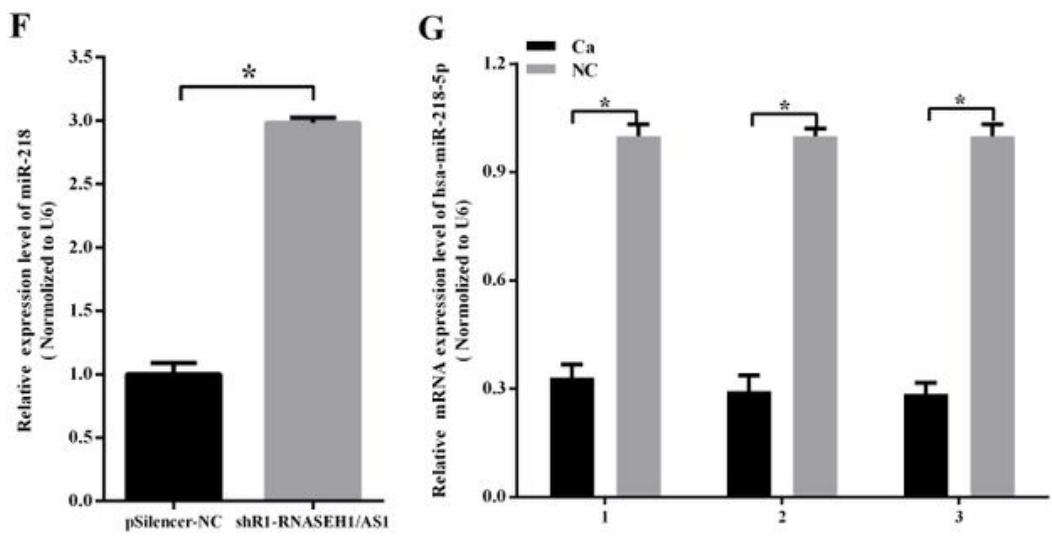

\section{Figure 1}

The IncRNA RNASEH1-AS1 is ceRNA for miR-218-5p. (A) RNASEH1-AS1 expression in cancer and normal tissue samples determined by screening StarBase V3.0. RNASEH1-AS1 was obviously upregulated in cancer samples compared to normal tissue samples (p囚0.05). (B) RNASEH1-AS1 expression in three pairs of clinical tissue samples analysed using qRT-PCR. The relative foldchange in RNASEH1-AS1 expression was calculated using $\beta$-actin as the internal control. The $Y$ axis presents the means $\pm S . D$. of three separate 


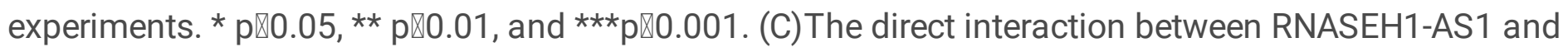
miR-218-5p was confirmed using a luciferase report assay. (D) The effect of miR-218-5p on the level of the endogenous IncRNA RNASEH1-AS1 determined using qRT-PCR. (E) The interfering effect of shRNASEH1-AS1 plasmid. (F) The effect of the IncRNA RNASEH1-AS1 on the endogenous miR-218-5p level determined using qRT-PCR. (G) Expression of miR-218-5p in three pairs of clinical tissue samples measured using qRT-PCR.

A

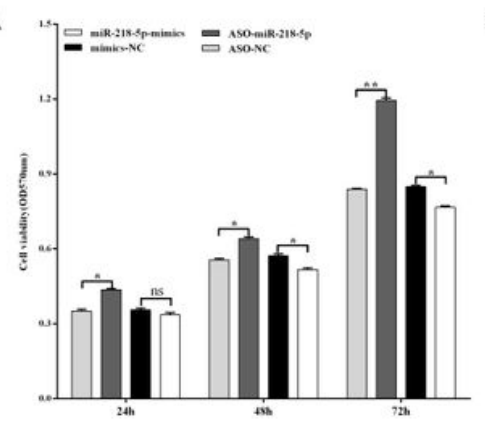

B

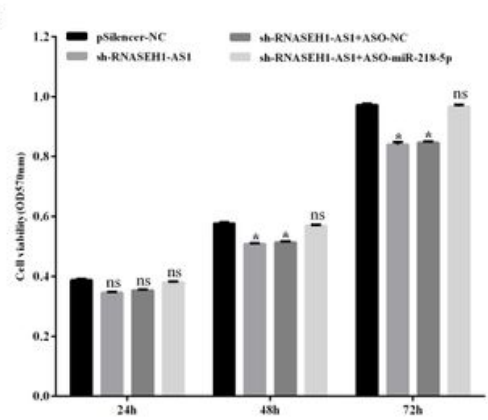

C

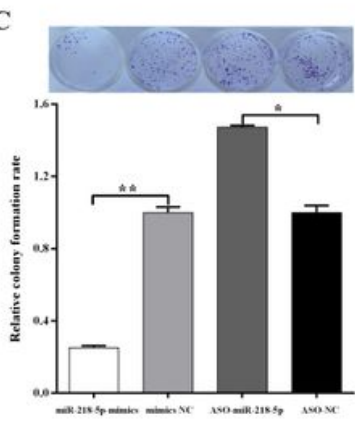

D

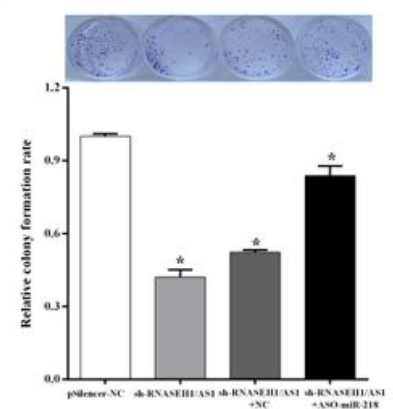

E

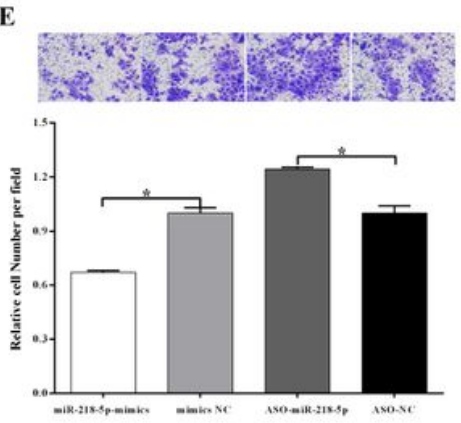

F

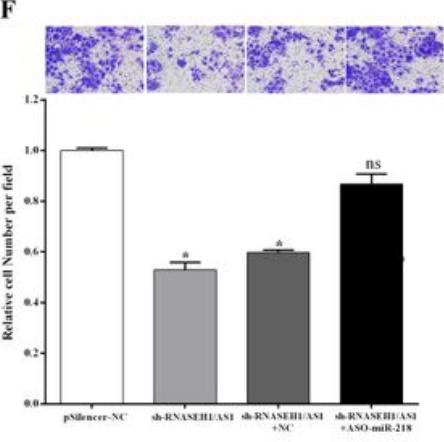

G
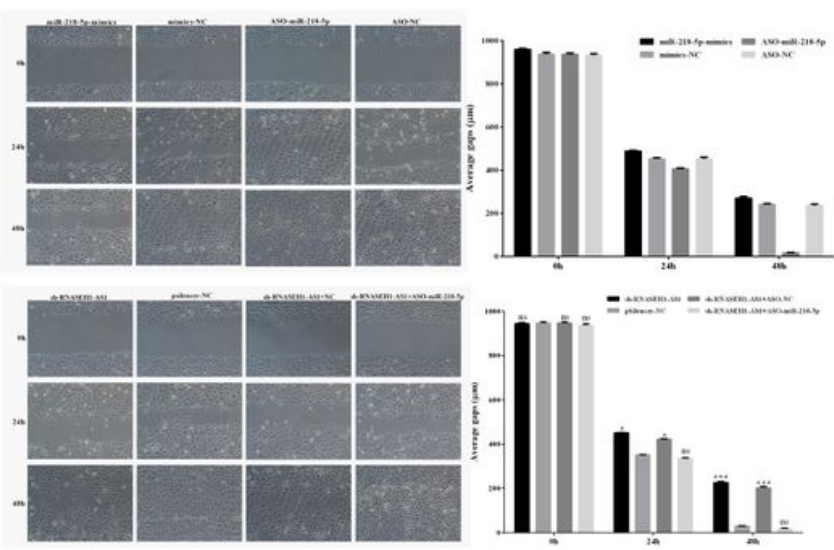

Figure 2

The functions of miR-218-5p and RNASEH1-AS1 in LUSC cells. ( $A$ and B) Results of the MTT assay performed in $\mathrm{NCl}-\mathrm{H} 520$ cells. ( $\mathrm{C}$ and D) Results of the colony formation assay performed in $\mathrm{NCl}-\mathrm{H} 520$ cells. (E and F) Results of the transwell invasion assay conducted with NCl-H520 cells. (G) Results of the wound-healing assay performed using $\mathrm{NCl}-\mathrm{H} 520$ cells. 


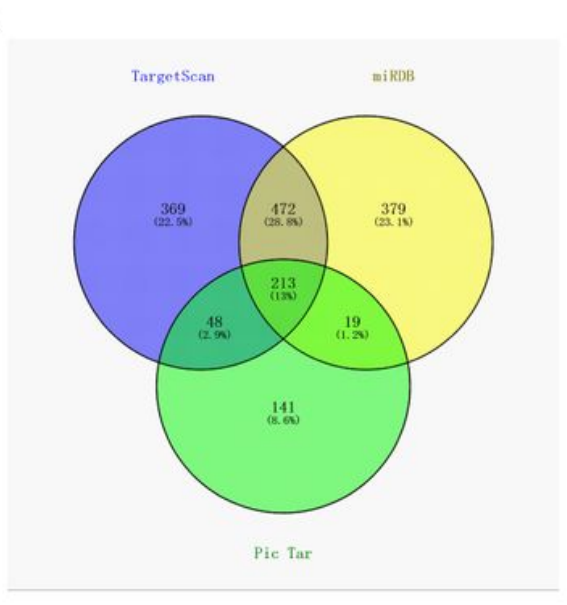

B
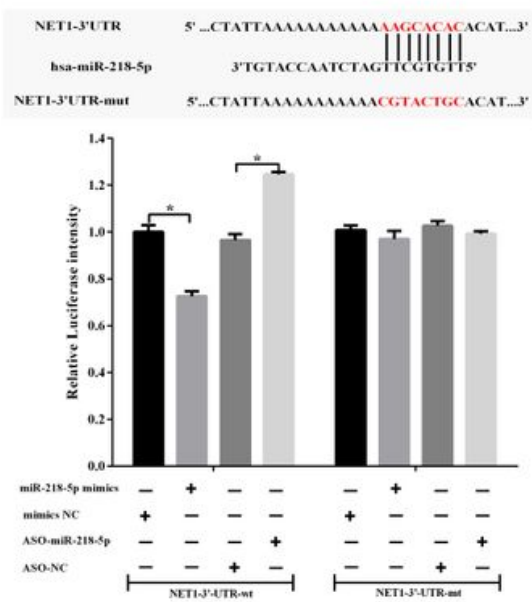

C

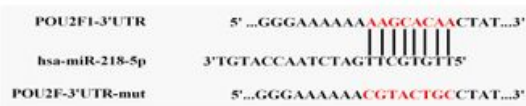

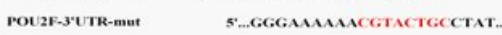

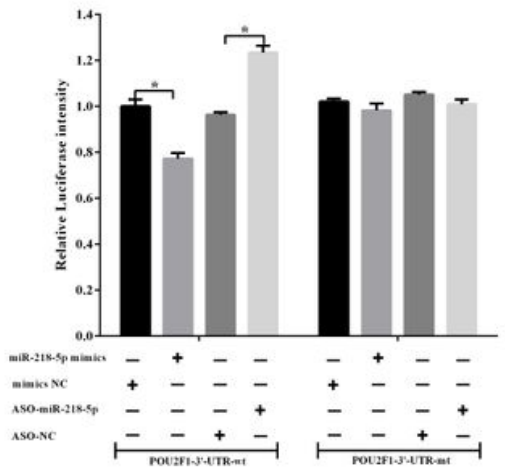

G

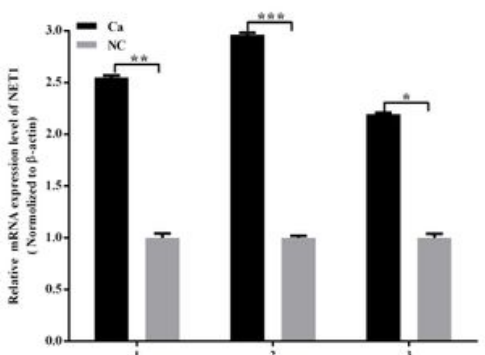

Figure 3

miR-218-5p directly targets NET1 and POU2F1. (A) Targets predicted by Target Scan, miRDB and PicTar. (B) The direct interaction between miR-218-5p and NET1 was confirmed by conducting a luciferase reporter assay. (C) The direct interaction between miR-218-5p and POU2F1 was confirmed by performing a luciferase reporter assay. (D) The effect of miR-218-5p on the expression of the endogenous NET1 and POU2F1 mRNAswas determined using qRT-PCR. (E and F) The effect of miR-218-5p on levels of the endogenous NET1 and POU2F1 proteinswere determined using western blotting. The full-length blots are presented in Supplementary Figure S1 and Supplementary Figure S2. (G) NET1 expression in three pairs of clinical tissue samples measured using qRT-PCR. 
A

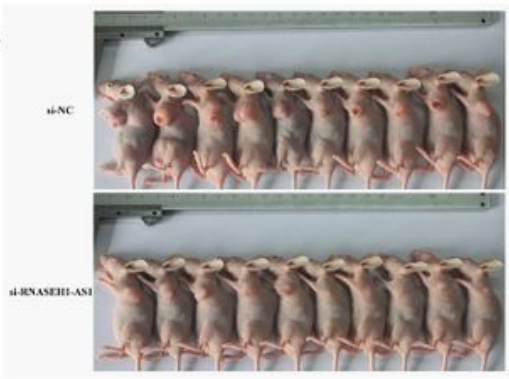

C

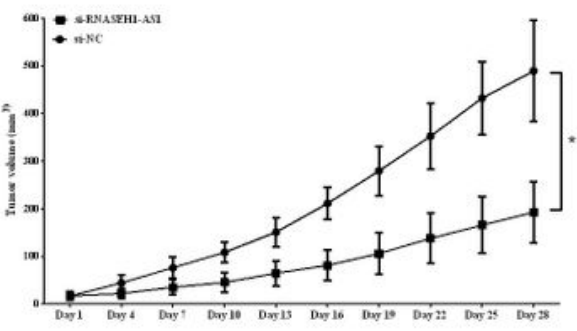

D

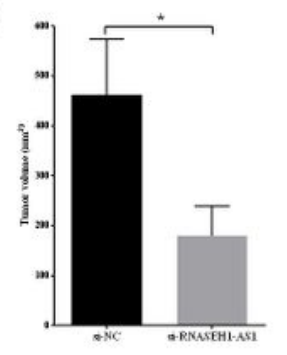

E

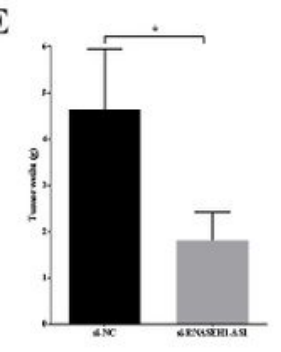

F

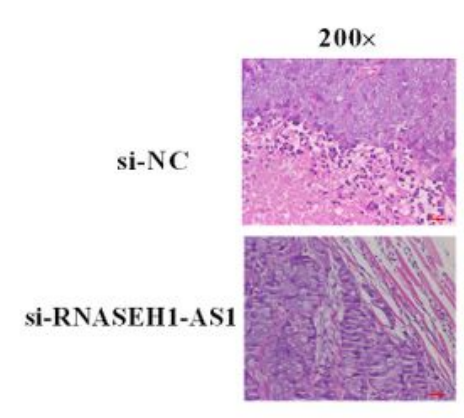

G

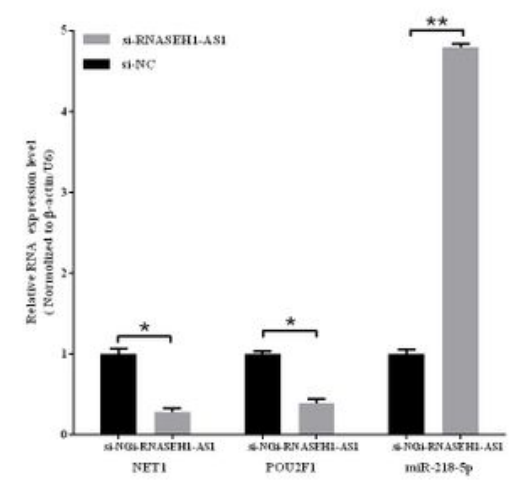

B

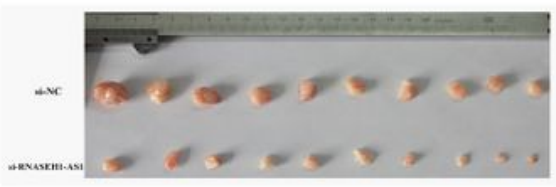

$$
\text { . }
$$


218-5p mRNA levels in xenograft tumoursdetected using qRT-PCR. $(\mathrm{H})$ western blots showing levels of the NET1 and POU2F1 proteinsin xenograft The full-length blots are presented in Supplementary Figure S3.

A

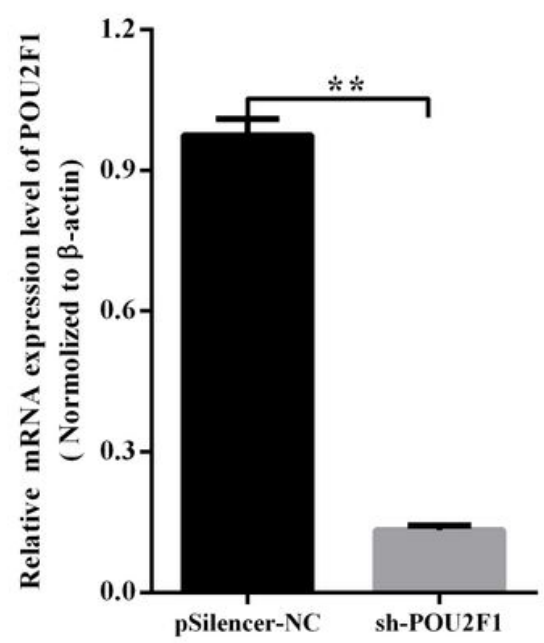

C

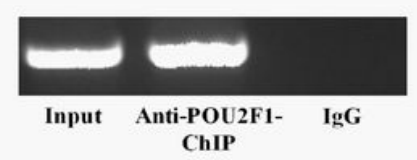

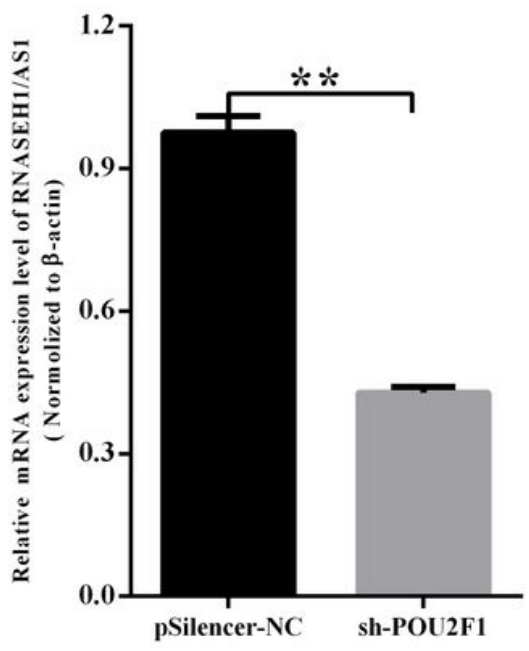

B

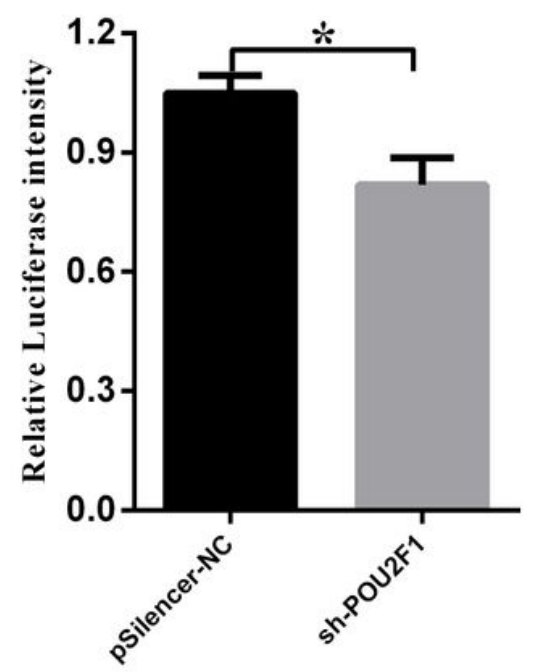

D

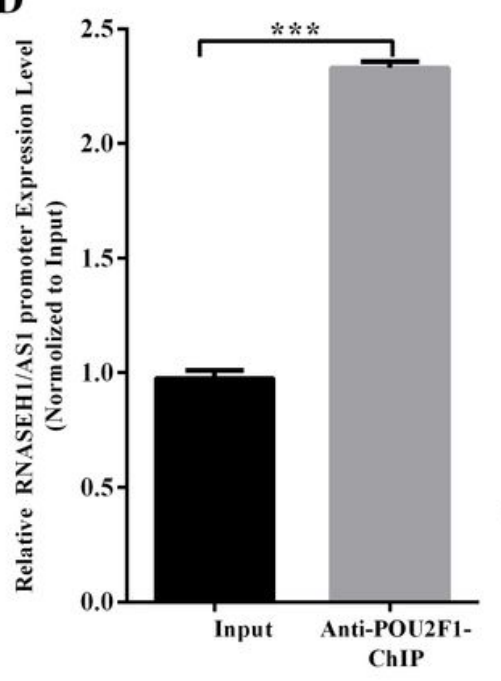

$\mathbf{E}$

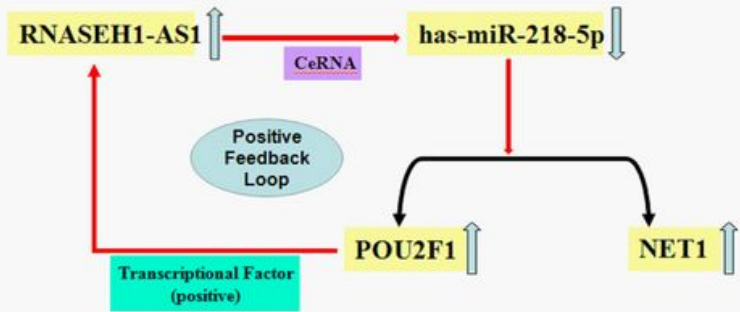

\section{Figure 5}

POU2F1 is a transcription factor for RNASEH1-AS1. (A) The interfering effect of the sh-POU2F1 plasmid. Decreased level of RNASEH1-AS1 after POU2F1 knockdown. (B) Decreased activity of the RNASEH1-AS1 promoter after knockdown of POU2F1. ( $C$ and D) The positive relationship between POU2F1 and RNASEH1-AS1 expression in the CHIP assay. The quantity of the input group was defined as 1 , and the fold change (2.30) of the anti-POU2F1-ChIP group was calculated. The full-length blots are presented in Supplementary Figure S4. (E) Summary of the results from this study.

\section{Supplementary Files}

This is a list of supplementary files associated with this preprint. Click to download. 
- NC3RsARRIVEGuidelinesChecklist2014.docx

- miR2185pSupplementarylnformation.docx 